\title{
Validation of Attitude and Heading Reference System and Microsoft Kinect for Continuous Measurement of Cervical Range of Motion Compared to the Optical Motion Capture System
}

\author{
Young Seop Song, $\mathrm{MD}^{1,2}$, Kyung Yong Yang, $\mathrm{MS}^{3}$, Kibum Youn, $\mathrm{MS}^{3}$, Chiyul Yoon, $\mathrm{PhD}^{2}$, \\ Jiwoon Yeom, $\mathrm{MD}^{1}$, Hyeoncheol Hwang, $\mathrm{MD}^{1,2}$, Jehee Lee, $\mathrm{PhD}^{3}$, Keewon Kim, MD, $\mathrm{MS}^{1,2}$
}

\begin{abstract}
${ }^{1}$ Department of Rehabilitation Medicine, Seoul National University Hospital, Seoul National University College of Medicine, Seoul; ${ }^{2}$ Interdisciplinary Program of Bioengineering, Graduate School, Seoul National University, Seoul;

${ }^{3}$ School of Computer Science and Engineering, Seoul National University, Seoul, Korea
\end{abstract}

\begin{abstract}
Objective To compare optical motion capture system (MoCap), attitude and heading reference system (AHRS) sensor, and Microsoft Kinect for the continuous measurement of cervical range of motion (ROM).

Methods Fifteen healthy adult subjects were asked to sit in front of the Kinect camera with optical markers and AHRS sensors attached to the body in a room equipped with optical motion capture camera. Subjects were instructed to independently perform axial rotation followed by flexion/extension and lateral bending. Each movement was repeated 5 times while being measured simultaneously with 3 devices. Using the MoCap system as the gold standard, the validity of AHRS and Kinect for measurement of cervical ROM was assessed by calculating correlation coefficient and Bland-Altman plot with 95\% limits of agreement (LoA).

Results MoCap and ARHS showed fair agreement $\left(95 \% \mathrm{LoA}<10^{\circ}\right)$, while MoCap and Kinect showed less favorable agreement $\left(95 \% \mathrm{LoA}>10^{\circ}\right)$ for measuring ROM in all directions. Intraclass correlation coefficient (ICC) values between MoCap and AHRS in $-40^{\circ}$ to $40^{\circ}$ range were excellent for flexion/extension and lateral bending (ICC $>0.9$ ). ICC values were also fair for axial rotation (ICC >0.8). ICC values between MoCap and Kinect system in $-40^{\circ}$ to $40^{\circ}$ range were fair for all motions.

Conclusion Our study showed feasibility of using AHRS to measure cervical ROM during continuous motion with an acceptable range of error. AHRS and Kinect system can also be used for continuous monitoring of flexion/ extension and lateral bending in ordinary range.
\end{abstract}

Keywords Joint range of motion, Neck, Sensor, AHRS, Kinect

Received June 1, 2015; Accepted September 21, 2015

Corresponding author: Keewon Kim

Department of Rehabilitation Medicine, Seoul National University Hospital, 101 Daehak-ro, Jongno-gu, Seoul 03080, Korea. Tel: +82-2-2072-0744, Fax: +82-2-743-7473, E-mail: KeewonKimM.D@gmail.com

ORCID: Young Seop Song (http://orcid.org/0000-0003-3503-1627); Kyung Yong Yang (http://orcid.org/0000-0003-2137-6018); Kibum Youn (http:// orcid.org/0000-0002-4061-3157); Chiyul Yoon (http://orcid.org/0000-0003-0754-058X); Jiwoon Yeom (http://orcid.org/0000-0002-1431-9907); Hyeoncheol Hwang (http://orcid.org/0000-0003-4119-2742); Jehee Lee (http://orcid.org/0000-0003-1023-1868); Keewon Kim (http://orcid.org/00000001-6597-578X).

(c) This is an open-access article distributed under the terms of the Creative Commons Attribution Non-Commercial License (http://creativecommons.org/ licenses/by-nc/4.0) which permits unrestricted noncommercial use, distribution, and reproduction in any medium, provided the original work is properly cited. Copyright @ 2016 by Korean Academy of Rehabilitation Medicine 


\section{INTRODUCTION}

Cervical range of motion (ROM) has been measured in many disorders of the cervical spine. It has been conventionally measured using a goniometer or cervical range of motion device (CROM; Performance Attainment Associates, St. Paul, MN, USA). Although these conventional methods are accurate enough to measure static state [1], continuous monitoring during dynamic motion is impossible. Therefore, those patients with dynamic cervical disorders such as cervical dystonia who would benefit from objective continuous cervical monitoring for evaluating disease severity and treatment outcome are evaluated with subjective scales $[2,3]$.

Recent studies have shown promising results in measuring dynamic cervical motion using methods such as in vitro measurement using implantable micro sensors on spine model [4] and in vivo measurements using fluoroscopy [5], biplane radiograph [6], and 3D computed tomography [7]. However, these devices are not clinically applicable for long duration due to radiation hazard, invasiveness, and the need of bulky imaging devices. To overcome these shortcomings, various new devices not conventionally considered as medical devices have been used in attempt to measure human motions. Microsoft Kinect (Kinect) is one of the most attractive options on the market. It uses in-depth camera information and probabilistic model for recognizing body part in real time [8]. It doesn't require any additional equipment that may interfere with motions. Acceptable accuracy $[9,10]$ and affordable price (US\$199) has made Kinect popular in various fields of research [11-14]. Another device of interest is the recently developed attitude and heading reference system (AHRS) based on microelectromechanical systems (MEMS) technology. It has benefit of small in size and light in weight. It is becoming popular for various applications such as underwater navigation, aircraft guidance control, and human motion capture [15-17].

However, the validity of AHRS and Kinect for measuring cervical ROM is currently unknown. The objective of this study was to compare AHRS and Kinect to the optical motion capture (MoCap) system that has been used extensively in researches for continuously obtaining human motion data without loss of precision [18] and for continuous measurement of cervical ROM [19].

\section{MATERIALS AND METHODS}

\section{Subjects}

Fifteen healthy adult subjects without neck pain or limitation in cervical motions were recruited in this study (12 males and 3 females; age, $27.7 \pm 4.6$ years; height, $171.1 \pm 5.7 \mathrm{~cm})$. The study protocol in compliance with the Declaration of Helsinki was approved by the Institutional Review Board of Seoul National University Hospital (No. H-1210-007-431). Informed consent was obtained from all subjects after the details of the study were explained.

\section{Equipment}

\section{Optical motion capture system}

Vicon VCam system (Vicon, Oxford, UK) composed of 14 cameras with 0.3 megapixel resolution was used. This system can perform motion capture at up to 120 frames per second (fps). In this study, motion capture was done in $30 \mathrm{fps}$ for convenient comparison with other interfaces.

\section{Wireless AHRS (EBIMU-9DOF, E2BOX, Seoul, Korea)}

The AHRS sensor was composed of a 3-axis gyroscope, a 3-axis acceleration sensor, and a 3-axis geomagnetic sensor. It was $15 \mathrm{~mm} \times 23.5 \mathrm{~mm}$ in size. It could transmit absolute angle of the sensor data wirelessly over Wi-Fi. Two sensors (one placed horizontally at the cranial vertex and the other one placed horizontally over the xiphoid process with a custom bracket) were used to calculate the relative motion of the head to the body.

Kinect and Kinect software development kit (Microsoft Corp., Bellevue, WA, USA)

Microsoft Kinect was used to measure the distance between the camera and the object in $320 \times 240$ resolution using infrared. An adoptable depth range was $40-450 \mathrm{~cm}$. Horizontal field of view was $57^{\circ}$. Vertical field of view was $43^{\circ}$.

Kinect software development kit (SDK) provided the tool and application program interface (API). Module for face recognition and body posture recognition were included in the Kinect SDK.

\section{Data collection}

Subjects were placed in a room equipped with optical motion capture cameras and the Kinect. Optical mark- 
ers were placed as described in the Vicon's manual (four markers on the head, five markers around the body, and a pair of markers on each side of shoulder, arm, and elbow). AHRS sensors were placed at the cranial vertex and over the xiphoid process to calculate the difference between them to minimize the effect of trunk movement (Fig. 1). Custom bracket was used to attach the AHRS sensors on the xiphoid process in similar horizontal plane with AHRS sensor on the cranial vertex to minimize measurement error from drift effect. Subjects were seated 100 $\mathrm{cm}$ in front of Kinect. They were instructed to perform independent movements in 3 axis (each repeated 5 times). Axial rotation to the preset point at approximately $50^{\circ}$ on each side followed by flexion/extension to the preset point at approximately $35^{\circ}$ on each side and lateral bending to their maximal range.

\section{Data analysis}

Data were collected in form of 3D coordinates for MoCap and Kinect or in the form of angle for AHRS. To convert 3D coordinates to angles, relationship of head, neck, and trunk was modeled as an articulated body (two rigid bodies connected by a ball joint) that could be expressed in chain of special Euclidean group matrix (SE(3)). For clinical interpretation, each component of the rotation of the ball joint was matched to flexion/extension, lateral

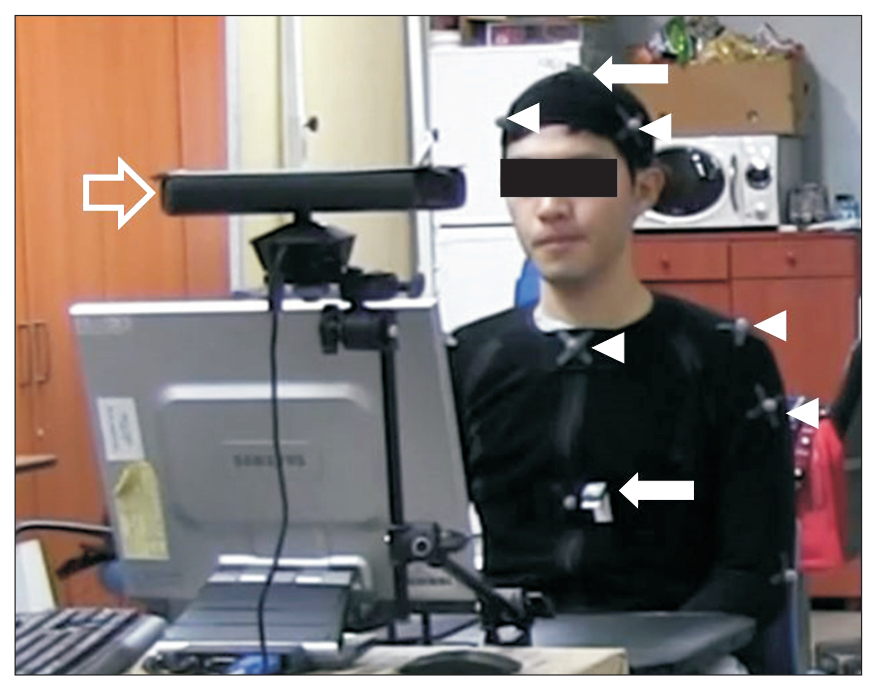

Fig. 1. Photograph of a subject wearing the attitude and heading reference system (AHRS) and optical marker in front of the Kinect for simultaneous measurement (white arrow, AHRS; void arrow, Kinect; arrowhead, optical marker). bending, or axial rotation. An expanded mathematical description of the conversion is provided in the Supplementary materials available with the full-text of this article at http://www.e-arm.org. To minimize the effect of different input latency of each interface for comparison, data were linearly interpolated by matching peak values.

\section{Statistical comparison}

Correlation (Pearson r) between each sensor and MoCap for the measurement of ROM conducted in this experiment was evaulated. The level of agreement was verified using the Bland-Altman plot with 95\% limits of agreement (LoA). Statistical analysis was computed with MATLAB (MathWorks, Natick, MA, USA). To show accuracy of each system for monitoring continuous motion, intraclass correlation coefficients (ICC) between each sensor and MoCap for every $10^{\circ}$ of measurement made by MoCap from $-40^{\circ}$ to $40^{\circ}$ range were computed using SPSS (SPSS Inc., Chicago, IL, USA). The range of $-40^{\circ}$ to $40^{\circ}$ was chosen considering that Kinect's face detection algorithm frequently failed when part of the face disappeared from the camera's view. Box plot of difference in measurements between MoCap and AHRS $(\Delta A)$ or between MoCap and Kinect $(\Delta K)$ for every $10^{\circ}$ of measurement made by MoCap in $-40^{\circ}$ to $40^{\circ}$ range was drawn.

\section{RESULTS}

MoCap and ARHS showed fair agreement (95\% LoA $<10^{\circ}$ ) for measuring ROM in all directions. MoCap and Kinect system showed less favorable agreement (95\% LoA $\left.>10^{\circ}\right)$ with negative average difference $\left(-7^{\circ}\right.$ to $\left.-7.5^{\circ}\right)$ for measuring ROM in all directions (Table 1, Fig. 2). Distribution of difference in measurement between each sensor and MoCap for every $10^{\circ}$ of measurement by MoCap is shown in Fig. 3. Axial rotation showed large measurement error distribution in both sensors and absolute measurement difference at the end of rotation. It was as high as $12.82^{\circ}$ for AHRS $\left(4.70^{\circ} \pm 3.04^{\circ}\right)$ and $14.25^{\circ}$ for Kinect $\left(4.75^{\circ} \pm 3.28^{\circ}\right)$. ICC values between MoCap and AHRS in $-40^{\circ}$ to $40^{\circ}$ range were excellent for flexion/extension and lateral bending (ICC >0.9). ICC values were fair for axial rotation (ICC $>0.8$ ). ICC values between MoCap and Kinect system in $-40^{\circ}$ to $40^{\circ}$ range were fair for all motions (ICC>0.8) (Table 2). 
Table 1. Comparison of range of motion measurement between different devices

\begin{tabular}{llcccc}
\hline \multirow{2}{*}{ Device } & \multirow{2}{*}{ Motion } & \multirow{2}{*}{ Pearson $\mathbf{r}$} & \multirow{2}{*}{ Mean difference $\left(^{\circ}\right)$} & \multicolumn{2}{c}{ 95\% LoA $\left(^{\circ}\right)$} \\
\cline { 5 - 6 } MoCap-AHRS & Flexion/extension & 0.96 & 2.4 & -4.4 & Upper \\
& Lateral bending & 0.97 & 2.7 & -2.8 & 9.1 \\
& Rotation & 0.96 & -2.8 & -8.5 & 8.1 \\
\multirow{2}{*}{ MoCap-Kinect } & Flexion/extension & 0.96 & -7 & -12.0 & -1.7 \\
& Lateral bending & 0.84 & -7.4 & -18.0 & 3.0 \\
& Rotation & 0.92 & -7.5 & -14.0 & -0.6 \\
\hline
\end{tabular}

LoA, limits of agreement; MoCap, motion capture; AHRS, attitude and heading reference system.

MoCap and AHRS-flexion/extension
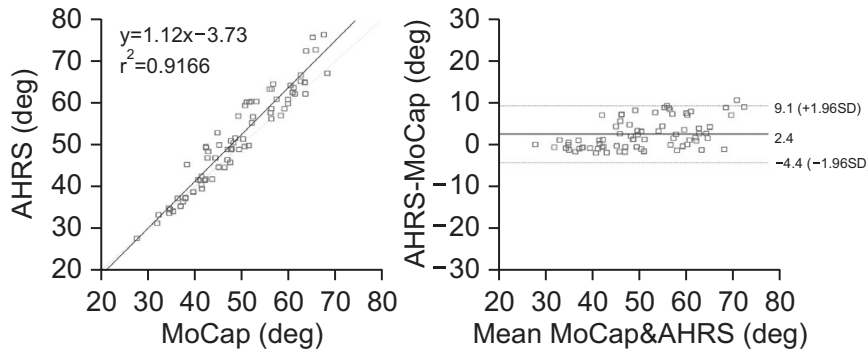

MoCap and AHRS-lateral bending
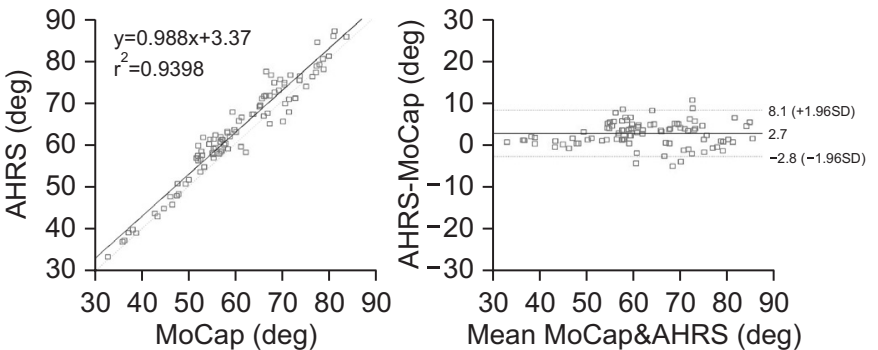

MoCap and AHRS-rotation
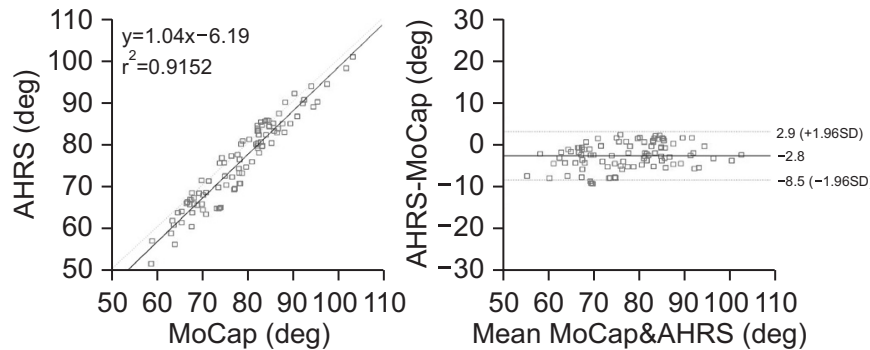

MoCap and Kinect-flexion/extension
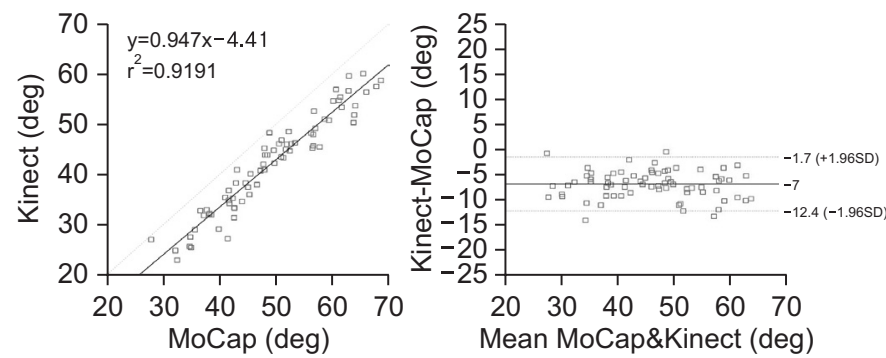

MoCap and Kinect-lateral bending
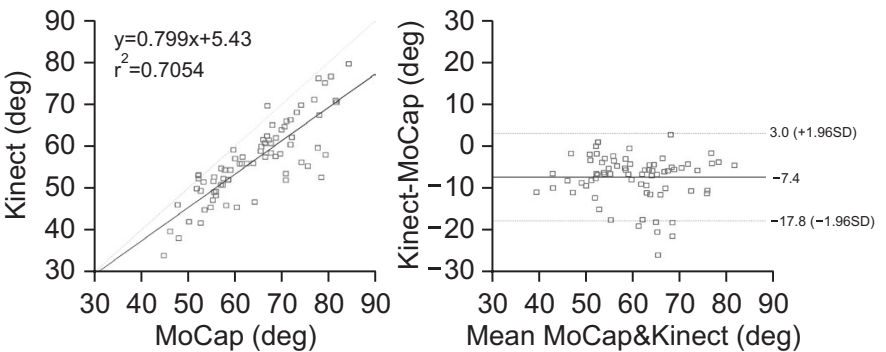

MoCap and Kinect-rotation
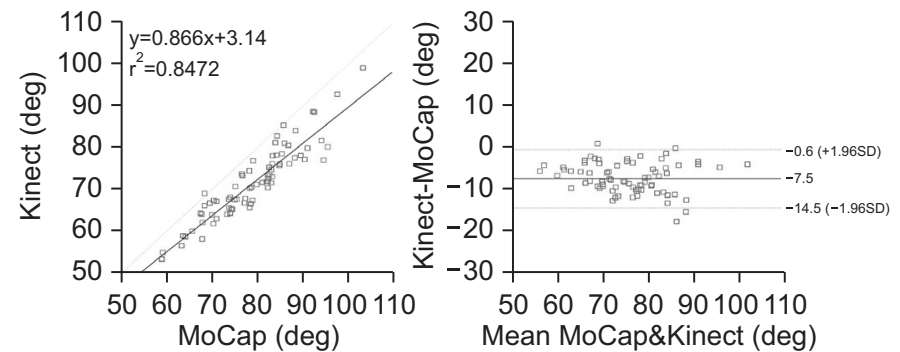

Fig. 2. Correlation and Bland-Altman plot of agreement between each sensor and optical motion capture (MoCap) system for measurement of maximal range of motion. AHRS, attitude and heading reference system.

\section{DISCUSSION}

This is the first study to investigate the feasibility of the AHRS and the Kinect system compared to MoCap for monitoring cervical motion. Our results showed that AHRS could be used to measure ROM during continu- ous movement in all directions with acceptable range of measurement error.

Kinect showed larger measurement error. It mostly had smaller measurement values compared to MoCap. For monitoring continuous motion, AHRS showed excellent correlation with MoCap while Kinect showed good cor- 

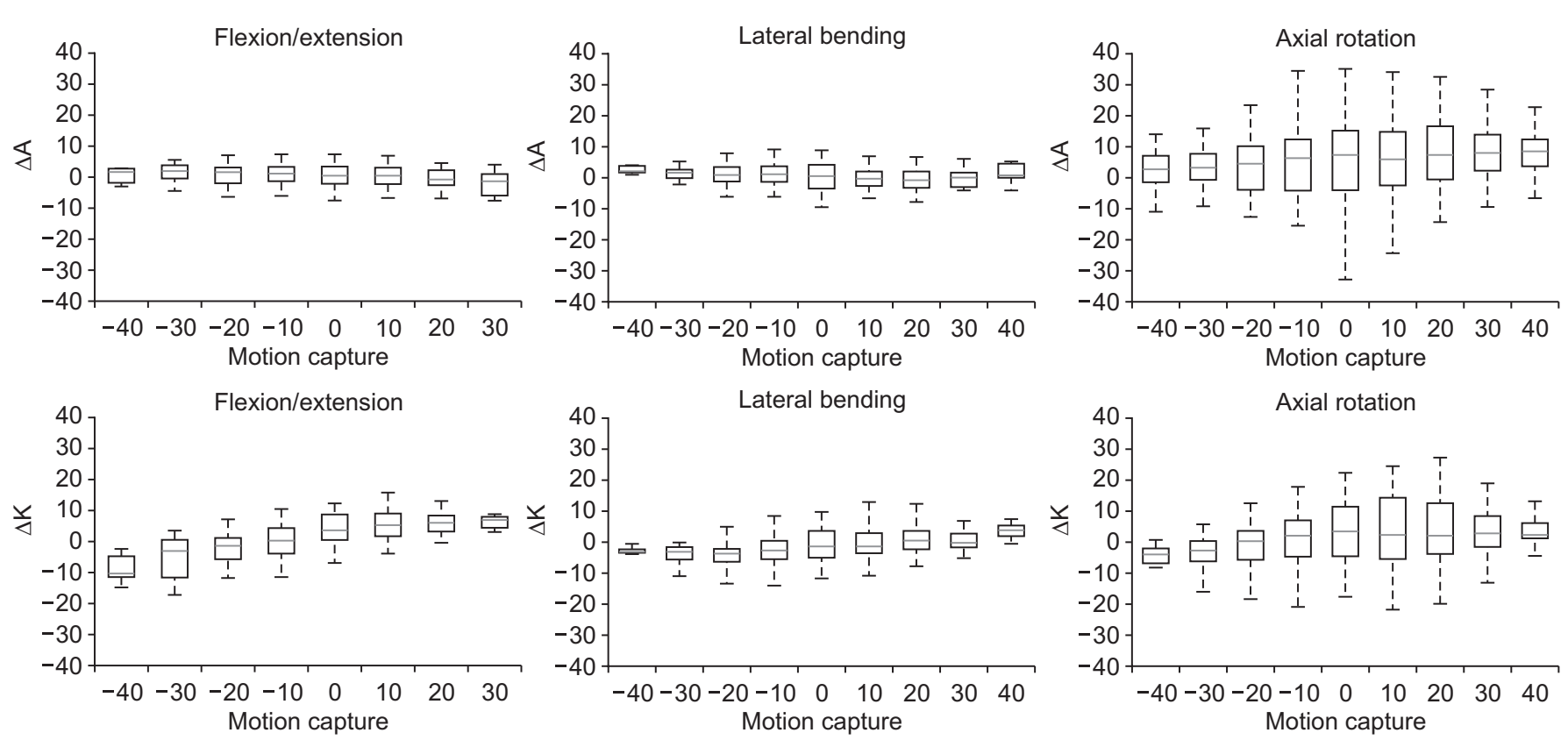

Fig. 3. Box plot showing distribution of measurement error of the AHRS and Kinect for every $10^{\circ}$ of measurement by optical motion capture system (red band inside the box, median; bottom and top of the box, the first and third quartiles). The lowest datum was within 0.953 interquartile range (IQR) of the lower quartile. The highest datum was within $0.953 \mathrm{IQR}$ of the upper quartile.

Table 2. ICC between each sensor and MoCap in $-40^{\circ}$ to $40^{\circ}$ range

\begin{tabular}{lcc}
\hline \multirow{2}{*}{\multicolumn{1}{c}{ Motion }} & \multicolumn{2}{c}{ ICC (95\% CI) } \\
\cline { 2 - 3 } & MoCap-AHRS & MoCap-Kinect \\
\hline Flexion/extension & $0.98(0.97-0.98)$ & $0.82(0.78-0.85)$ \\
Lateral bending & $0.92(0.91-0.93)$ & $0.84(0.79-0.88)$ \\
Axial rotation & $0.87(0.81-0.91)$ & $0.85(0.83-0.86)$ \\
\hline
\end{tabular}

ICC, intraclass correlation coefficient; CI, confidence interval; MoCap, motion capture; AHRS, attitude and heading reference system.

relation with MoCap for flexion/extension and lateral bending in $-40^{\circ}$ to $40^{\circ}$ range with larger measurement errors for axial rotation in both devices. However, there is no clear cutoff value for interpreting the LoA or correlation coefficient to decide whether a device is valid or useful compared to the gold standard [20]. Instead, the validity of a device depends on clinical setting of the usage and how much error can be tolerated.

These new devices have larger measurement errors compared to the results obtained from fluoroscopy or Xrays [5-7]. This error may limit sensor efficacy. Accuracy of AHRS is known to be affected by time, velocity, acceleration, and magnetic distortion. To minimize these measurement errors, various algorithms and filters have been studied [21-24]. In our study, peak angular velocity and acceleration were larger in axial rotation than those in flexion/extension and lateral bending. This might be the reason for the larger measurement error in axial rotation. Accuracy of Kinect can be affected by distance between subject and the camera and recognition precision. In our study, subjects were placed $100 \mathrm{~cm}$ in front of the camera with anticipated measurement error of $<0.03 \mathrm{~cm}$ [10]. However, face detection algorithm failed to accurately detect the face in extreme angles. In these angles, simultaneous exposure of tip of the mandible and middle of forehead for appropriate facial recognition were not satisfied. Other factor that might have contributed to negative systemic error shown on Kinect measurement could be inadequate initialization or error in the process of converting coordinates to angles.

During data analysis, we noted that lateral bending, flexion/extension, and axial rotation were not independent motions. Small amplitude of lateral bending with flexion/extension accompanied axial rotation has been shown in previous study results $[25,26]$. This phenomenon indicates that the axis of the axial rotation can tilt during axial movement in relation to the body. Moreover, 
the axis of each cervical motion is not static. Instead, it is rather complex and dynamic $[6,27]$. A multi joint model rather than a single ball and socket joint model is needed to describe it mathematically. Another limitation of our study is the small number of participants with limited range in age distribution. Age has been found to have negative correlation with active cervical ROM [28,29]. It might be more suitable to apply these devices in older populations.

In conclusion, our study showed the feasibility of using AHRS to measure cervical ROM during continuous motion with an acceptable range of error. AHRS and Kinect system can also be used for continuous monitoring of flexion/extension and lateral bending in ordinary ROM. These systems are ubiquitous, they have minimal contact with the body and relatively low cost compared to MoCap. Therefore, they can be used to monitor various diseases and activities in ordinary range.

\section{CONFLICT OF INTEREST}

No potential conflict of interest relevant to this article was reported.

\section{SUPPLEMENTARY MATERIALS}

Supplementary materials can be found via http:// dx.doi.org/10.5535/arm.2016.40.4.568. Modelling cervical motion as a ball joint.

\section{REFERENCES}

1. Youdas JW, Carey JR, Garrett TR. Reliability of measurements of cervical spine range of motion: comparison of three methods. Phys Ther 1991;71:98-104

2. Cano SJ, Hobart JC, Fitzpatrick R, Bhatia K, Thompson AJ, Warner TT. Patient-based outcomes of cervical dystonia: a review of rating scales. Mov Disord 2004; 19:1054-9.

3. Jost WH, Hefter H, Stenner A, Reichel G. Rating scales for cervical dystonia: a critical evaluation of tools for outcome assessment of botulinum toxin therapy. J Neural Transm (Vienna) 2013;120:487-96.

4. Mularski S, Picht T, Kuehn B, Kombos T, Brock M, Suess O. Real-time tracking of vertebral body movement with implantable reference microsensors. Com- put Aided Surg 2006;11:137-46.

5. Bifulco P, Cesarelli M, Romano M, Fratini A, Sansone M. Measurement of intervertebral cervical motion by means of dynamic x-ray image processing and data interpolation. Int J Biomed Imaging 2013;2013:152920.

6. Haque MA, Anderst W, Tashman S, Marai GE. Hierarchical model-based tracking of cervical vertebrae from dynamic biplane radiographs. Med Eng Phys 2013;35:994-1004.

7. Anderst WJ, Baillargeon E, Donaldson WF 3rd, Lee JY, Kang JD. Validation of a noninvasive technique to precisely measure in vivo three-dimensional cervical spine movement. Spine (Phila Pa 1976) 2011;36:E393400.

8. Shotton J, Sharp T, Kipman A, Fitzgibbon A, Finocchio $\mathrm{M}$, Blake A, et al. Real-time human pose recognition in parts from single depth images. Commun ACM 2013;56:116-24.

9. Schmitz A, Ye M, Shapiro R, Yang R, Noehren B. Accuracy and repeatability of joint angles measured using a single camera markerless motion capture system. J Biomech 2014;47:587-91.

10. Khoshelham K, Elberink SO. Accuracy and resolution of Kinect depth data for indoor mapping applications. Sensors (Basel) 2012;12:1437-54.

11. Zhang Z. Microsoft kinect sensor and its effect. IEEE Multimed 2012;19:4-10.

12. Fern'ndez-Baena A, Susin A, Lligadas X. Biomechanical validation of upper-body and lower-body joint movements of kinect motion capture data for rehabilitation treatments. Proceedings of 2012 4th International Conference on Intelligent Networking and Collaborative Systems (INCoS); 2012 Sep 19-21; Bucharest, Romania. p. 656-61.

13. Oikonomidis I, Kyriazis N, Argyros AA. Efficient model-based 3D tracking of hand articulations using Kinect. Proceedings of the British Machine Vision Conference (BMVC); 2011 Aug 29-Sep 2; Dundee, UK. p. 1-11.

14. Chang YJ, Chen SF, Huang JD. A Kinect-based system for physical rehabilitation: a pilot study for young adults with motor disabilities. Res Dev Disabil 2011;32:2566-70.

15. Wendel J, Meister O, Schlaile C, Trommer GF. An integrated GPS/MEMS-IMU navigation system for an autonomous helicopter. Aerosp Sci Technol 2006;10:527- 
33.

16. Armstrong B, Wolbrecht E, Edwards DB. AUV navigation in the presence of a magnetic disturbance with an extended Kalman filter. Proceedings of the OCEANS 2010 IEEE; 2010 May 24-27; Sydney, Australia. p. 1-6.

17. Kim H, Shin SH, Kim JK, Park YJ, Oh HS, Park YB. Cervical coupling motion characteristics in healthy people using a wireless inertial measurement unit. Evid Based Complement Alternat Med 2013;2013:570428.

18. Windolf M, Gotzen N, Morlock M. Systematic accuracy and precision analysis of video motion capturing systems: exemplified on the Vicon-460 system. J Biomech 2008;41:2776-80.

19. Henmi S, Yonenobu K, Masatomi T, Oda K. A biomechanical study of activities of daily living using neck and upper limbs with an optical three-dimensional motion analysis system. Mod Rheumatol 2006;16:28993.

20. Lexell JE, Downham DY. How to assess the reliability of measurements in rehabilitation. Am J Phys Med Rehabil 2005;84:719-23.

21. Lebel K, Boissy P, Hamel M, Duval C. Inertial measures of motion for clinical biomechanics: comparative assessment of accuracy under controlled conditions - effect of velocity. PLoS One 2013;8:e79945.

22. Lebel K, Boissy P, Hamel M, Duval C. Inertial measures of motion for clinical biomechanics: comparative assessment of accuracy under controlled conditions - changes in accuracy over time. PLoS One 2015; 10:e0118361.

23. Yadav N, Bleakley C. Accurate orientation estimation using AHRS under conditions of magnetic distortion. Sensors (Basel) 2014;14:20008-24.

24. Luinge HJ, Veltink PH. Inclination measurement of human movement using a 3-D accelerometer with autocalibration. IEEE Trans Neural Syst Rehabil Eng 2004;12:112-21.

25. Ishii T, Mukai Y, Hosono N, Sakaura H, Nakajima Y, Sato $\mathrm{Y}$, et al. Kinematics of the upper cervical spine in rotation: in vivo three-dimensional analysis. Spine (Phila Pa 1976) 2004;29:E139-44.

26. Zhao X, Wu ZX, Han BJ, Yan YB, Zhang Y, Lei W. Three-dimensional analysis of cervical spine segmental motion in rotation. Arch Med Sci 2013;9:515-20.

27. Anderst W, Baillargeon E, Donaldson W, Lee J, Kang J. Motion path of the instant center of rotation in the cervical spine during in vivo dynamic flexion-extension: implications for artificial disc design and evaluation of motion quality after arthrodesis. Spine (Phila Pa 1976) 2013;38:E594-601.

28. Sforza C, Grassi G, Fragnito N, Turci M, Ferrario V. Three-dimensional analysis of active head and cervical spine range of motion: effect of age in healthy male subjects. Clin Biomech (Bristol, Avon) 2002;17:611-4.

29. Youdas JW, Garrett TR, Suman VJ, Bogard CL, Hallman HO, Carey JR. Normal range of motion of the cervical spine: an initial goniometric study. Phys Ther 1992;72:770-80. 